\title{
EL IMPACTO DEL PROYECTO EDUCATIVO INSTITUCIONAL DEL INPEC EN UN GRUPO DE MUJERES POSPENADAS
}

\author{
THE IMPACT OF THE INSTITUTIONAL EDUCATIONAL \\ PROJECT OF INPEC ON A GROUP OF POST- \\ CONVICTED WOMEN
}

Adriana Yisela López García*

\footnotetext{
* Magíster en Educación de la Universidad Surcolombiana. Inspector en Instituto Nacional Penitenciario INPEC.

nanalopez0916@gmail.com 0000-0003-1471-6037

Cómo citar este artículo: López, G. A. Y. (2021). El impacto del proyecto educativo institucional del INPEC en un grupo de mujeres pospenadas. Revista PACA 11, pp. 35-50.
}

Resumen: Este artículo de investigación expone los principales aspectos de la sistematización de experiencias: el impacto del proyecto educativo institucional del INPEC en un grupo de mujeres pospenadas, como medio para reflexionar sobre la relación de la educación penitenciaria y los procesos de resocialización. El objetivo general de este escrito es sistematizar una experiencia en el campo de la educación penitenciaria para visibilizar y reflexionar sobre los impactos del proyecto educativo del INPEC en la cualificación de un grupo de mujeres pospenadas. Se enfoca el tipo de investigación a través del paradigma sociocrítico que se caracteriza por una continua acciónreflexión-acción, desarrollado mediante el método de sistematización de experiencias educativas. Los principales hallazgos permiten ver que los modelos educativos penitenciarios están supeditados a una serie de factores que influyen en el diseño organizacional, el establecimiento, la administración y la gobernanza. Además, que las condiciones de vida en una prisión se encuentran entre los principales factores que determinan la potenciación del ser humano que cumple una condena; en ese sentido, la autoestima y la dignidad con las que se les trata en el centro penitenciario delimita la posibilidad del PPL de tener éxito en el proceso de reintegrarse a la sociedad como ciudadano respetuoso de la ley. 
Palabras clave: Educación penitenciaria, sistematización de experiencias, ambiente, prisión, mujer, CLEIS.

\begin{abstract}
This research article exposes the main aspects of the systematization of experiences: the impact of the institutional educational project of INPEC on a group of post-sentenced women, as a means of reflecting on the relationship between prison education and the processes of resocialization. The general objective of this writing is to systematize an experience in the field of prison education to make visible and reflect on the impacts of the INPEC educational project on the qualification of a group of post-sentenced women. The type of research is approached through the socio-critical paradigm that is characterized by a continuous action-reflection-action, developed through the method of systematizing educational experiences. The main findings allow us to see that prison educational models are subject to a series of factors that influence organizational design, establishment, administration and governance. In addition, that the living conditions in a prison are among the main factors that determine the empowerment of the human being serving a sentence in that sense of self-esteem and dignity with which they are treated in the penitentiary center delimits the possibility of the PPL of be successful in the process of reintegrating into society as a law-abiding citizen.
\end{abstract}

36 Keywords: Penitentiary education, systematization of experiences, environment, prison, woman, CLEIS.

\title{
Introducción
}

La educación en centro penitenciario ha sido propuesta desde el Ministerio de Educación Nacional como un medio para garantizar el derecho de la Persona Privada de la Libertad- PPL a recibir formación de calidad que mejore su calidad de vida, pues la reclusión, aunque es la sanción justificada por cometer un delito, no lleva implícita la privación adicional de los derechos fundamentales. En este sentido, la dirección de la educación del INPEC apunta a que los ciudadanos que han incurrido en delitos identifiquen que estas conductas no son válidas y legítimas, transformando su forma de pensar, su manera de ver la vida y redirigir su existencia para que puedan volver a la sociedad, orientar sus labores hacia el bien, construir otra estética de la vida, asumir otras maneras de interactuar al interior de la comunidad y constituirse en actores sociales de su desarrollo personal, familiar y colectivo. 
En efecto, la educación penitenciaria se presenta como un espacio en los centros de reclusión que apuntan no solo a formar al PPL, sino a capacitarlos en algunos aspectos y a cualificarlo en otros, aprovechando los espacios de la condena para darle un nuevo sentido al plan de vida del ciudadano privado de la libertad. Si bien los proyectos de educación en PPL se orientan desde los principios de cualquier proceso de educación, en Colombia son desarrollados en condiciones deficientes que se relacionan con el alto porcentaje de hacinamiento, la ausencia de espacios educativos propicios (aulas improvisadas), el deterioro del mobiliario requerido para orientar el proceso, la falta de material didáctico de apoyo para actividades de refuerzo, consulta y afianzamiento, además de la precariedad administrativa que claramente contraviene las necesidades propias de los ciclos CLEIS (ciclos lectivos especiales integrados).

En este escenario, el proceso educativo en centro de reclusión es difícil y complejo, pero también problemático si se piensa en el nuevo estatus social de la PPL cuando ha cumplido su condena y debe reincorporarse a las comunidades. Es justo ahí donde se evidencia la estigmatización social que enfrenta el pospenado, pero, además, es el escenario en el que se pone a prueba la tarea de resocialización que es crucial en el espacio carcelario, que no responde actualmente a las necesidades de formación que enfrenta el pospenado cuando cumple su condena.

A pesar de ello, es una realidad que quien recibe educación accede a un sin-número de beneficios personales: de participación, de pertenencia real a la sociedad, de elegir, de ser elegido, de construir un modelo de vida basado en la posibilidad de decidir, de ser libre frente al pensamiento y la decisión y, explícitamente, quien tiene educación puede en el sistema social acceder a mejores empleos, a seguir capacitándose, puede enseñar a otro pero, sobre todo, defender sus derechos, pues como dice el adagio popular "el conocimiento te da poder". Sin esa conciencia, la sociedad civil no priorizará educar a todos los ciudadanos con equidad, garantizando que en igualdad de condiciones todas las personas se formen según sus habilidades, gustos o afinidades, sino que la brecha social seguirá generando a través de la pobreza delincuencia y a través de la delincuencia mayor presión al desbordado sistema penitenciario de nuestro país que, dicho sea de paso, busca mediante el proceso educativo fundamentalmente la reinserción y la no reincidencia. Por los argumentos expresados anteriormente, se plantea la pregunta de 
investigación: ¿De qué manera la sistematización de experiencias en el campo de la educación penitenciaria permite visibilizar el impacto del proyecto educativo del INPEC en la cualificación y resignificación de las potencialidades de un grupo de mujeres pospenadas?

Es así como esta propuesta de sistematización de experiencias en el campo de la educación penitenciaria permite visibilizar el impacto del proyecto educativo del INPEC en la cualificación y resignificación de las potencialidades de un grupo de mujeres pospenadas del Establecimiento Penitenciario de Mediana Seguridad y Carcelario de Neiva- EPMSC. Asimismo, al ser un proyecto que se ha desarrollado hace décadas como medio para generar en el período de reclusión espacios para que las PPL se capaciten recibiendo beneficios en la rebaja de la pena, se busca suscitar reflexiones en torno a las modalidades educativas de centros penitenciarios, y no solamente informar a las personas acerca de los diversos aspectos que delimitan los espacios educativos sino lo significativo de los ambientes de aprendizaje, las clases como medio de resocialización y multiplicadores de acciones de convivencia.

En este contexto, se propone como objetivo general sistematizar una experiencia en el campo de la educación penitenciaria para visibilizar y reflexionar sobre los impactos del proyecto educativo del INPEC en la cualificación de un grupo de mujeres pospenadas, para ser desarrollado mediante tres acciones específicas: indagar sobre las intencionalidades y propósitos del Proyecto Educativo Institucional del Instituto Nacional Penitenciario en una práctica concebida para la población de internos del Establecimiento Penitenciario de Mediana Seguridad y Carcelario de Neiva- EPMSC; reconocer los impactos del proyecto de rehabilitación social para la población de internas del Establecimiento Penitenciario de Mediana Seguridad y Carcelario de Neiva- EPMSC en un grupo de mujeres pospenadas; y proponer reflexiones críticas sobre la educación penitenciaria y el proyecto educativo del INPEC, teniendo en cuenta las experiencias de población femenina pospenada del Establecimiento Penitenciario de Mediana Seguridad y Carcelario de Neiva- EPMSC.

Es aquí donde el ejercicio de indagación hace alusión a la situación de un segmento importante de ciudadanos a los que no se les ha garantizado el acceso a la educación, a oportunidades de trabajo o a la atención adecuada en materia de salud; también plantea cómo esta 
situación abominable de exclusión e inequidad los ha llevado a desarrollar mecanismos propios de supervivencia que se traducen en expresiones de criminalidad por las que posteriormente son privados de la libertad (Informe Anual del INPEC, 2019).

Además, se pretende observar desde la experiencia cómo es la realidad de una parte de la población en Colombia que convive con enormes brechas sociales, que se debate entre la pobreza, la falta de acceso a servicios públicos como acueducto y alcantarillado, las desigualdades en el reparto de recursos, los conflictos armados y la discriminación, entre otras tantas en materia de salud y educación.

En suma, este proyecto busca, a través de la reflexión, visibilizar el impacto del proyecto educativo del INPEC, reflejado en las experiencias de vida mujeres pospenadas que han podido, al cumplir condena, salir y vincularse activamente o si, por el contrario, el estigma del exconvicto ha sido determinante.

\section{Referentes teóricos}

Para abordar el problema que se ha descrito este apartado pretende analizar los fundamentos teóricos que subyacen en conceptos fundamentales como: la educación como una experiencia de sistematización educativa y como esta se ha realizado en PPL. Seguido a lo anterior se construye cómo es la experiencia de la mujer en prisión y en otro espacio del componente teórico se especifica qué es el currículo y cómo se propone esta figura en el proyecto educativo del INPEC para identificar cómo, mediante programas de educación penitenciaria, se puede afectar la condición del preso cuando pasa a la condición de pospenado.

La educación es un fenómeno que responde a las condiciones sociales específicas de la época en la que se busca definir. Siendo un proceso que depende del ser humano, es interpretado desde la subjetividad del observador, pero ha sido delimitado bajo los métodos, los ambientes, los espacios, la evaluación y los logros con el fin de generar puntos de encuentro dentro de la diversidad que significa aprender y enseñar. En los diferentes frentes a los que la educación apunta, en este proyecto se aborda la penitenciaria, concebida como los espacios de resocialización 
que garantizan los derechos fundamentales del PPL y contribuyen al sistema de rebaja de condena.

Acerca de la educación en prisiones, Español y Moreno (2014, p.8) indican que está reglamentada en Colombia por la Ley General de la Educación (1994); cuyo artículo 68 define el objetivo y ámbito de los programas de rehabilitación social para aquellas personas que necesitan reincorporarse a la sociedad. Y el artículo 69 establece que este tipo de educación requiere métodos didácticos, contenidos y procesos pedagógicos acordes con el contexto y sus educandos. Queda claro, en consecuencia, el interés del Estado por promover la educación para la rehabilitación social como servicio público integral. Y en cuanto a la educación vista por el INPEC, Español y Moreno (2014) expresan que en apartados específicos como el artículo 94 de la Ley 65 de 1993 se establece que el estudio y el trabajo son los dos campos fundamentales en los cuales se basa la resocialización.

Para Español y Moreno (2014) a los privados de la libertad se les debe garantizar el acceso a los programas de educación superior en instituciones educativas, no solo en los niveles de alfabetización. Sumado a este derecho se debe brindar a los PPL la posibilidad de desempeñarse en actividades de enseñanza, acorde con su perfil y capacidades.

En ese sentido, Rosas (2017) dice que el nuevo Modelo Educativo para el Sistema Penitenciario y Carcelario Colombiano nace de la necesidad de ofrecer una educación pertinente y adecuada al contexto de encierro de personas que han pasado por una experiencia relacionada con el delito. Se entiende que la educación de adultos requiere metodologías y currículos acordes con las relaciones sociales que se dan al interior de un centro penitenciario, como horarios, lugares de encuentro, relación con la guardia, los docentes, los internos monitores y las familias.

Además, el proceso educativo para Rosas (2017) se realiza a través del trabajo individual del interno, trabajo en grupo y un tercer momento de socialización de producciones. Se trabaja con el diseño de objetos de conocimiento que los internos desarrollan a través de preguntas e investigación "El acompañamiento de los monitores se realiza en los patios, celdas y área educativa del establecimiento" (p. 8). 
Luego Rosas (2017) identificó que, en la reclusión de mujeres de Bogotá, siguiendo las directrices planteadas por el INPEC, se han desarrollado procesos educativos en sus diferentes formas, como lo son la educación formal, informal y no formal; esto con el fin de contribuir en los procesos de resocialización. Teniendo en cuenta lo anterior, se ha identificado la falta escolaridad en la población reclusa, haciendo necesario comprender que el modelo educativo logra un cambio resocializador en el personal de internas de las cárceles, siendo entonces la educación una nueva opción en el proyecto de vida de las reclusas.

En suma, a lo anterior las mujeres encarceladas no solo son más proclives que los hombres a luchar contra el abuso de sustancias y los problemas de salud mental que se derivan del abuso físico o sexual; también tienden a ser un cuidador central de sus familias; además, por las hegemonías masculinas en otros aspectos sociales las mujeres tienden a estar en desventaja con respecto al hombre.

Desde otro punto de vista, en el entorno carcelario de Colombia la mayoría de las veces las mujeres luchan con historias de abuso de sustancias, violación, violencia, trauma infantil, violencia doméstica, enfermedades mentales y pobreza. A menudo, estos traumas se remontan a generaciones anteriores en las familias. Sin embargo, hay evidencias de que la educación ayuda a trascender el ciclo multigeneracional de encarcelamiento dentro de las familias que se pueden consultar en los antecedentes que se exponen en capítulos posteriores, donde varios autores como Sánchez, Rodríguez, Fondevila y Morad (2018) identifican que la mujer pospenada sí enfrenta marcadas diferencias con respecto al hombre pospenado.

En cuanto a la condición femenina de PPL, Schneider (2018) dice que en la órbita estatal se plantea la cuestión de género en las prisiones. Se observa que, así como las cárceles masculinas ofrecen propuestas de formación y trabajo históricamente ligadas al rol social del varón, lo mismo ocurre en el caso de las mujeres y son muy escasas las alternativas de enseñanza y actividades vinculadas a otras labores que no sean las tareas del hogar, lo que reduce a la mujer al histórico estigma de ser ama de casa, cuidar de los hijos y formarse en actividades tradicionalmente asignadas al género femenino. 
Además, Sánchez, Rodríguez, Fondevila y Morad (2018) en la publicación Mujeres y prisión en Colombia, desafíos para la política criminal desde un enfoque de género, indican que la mayoría de las mujeres PPL cumplen con un perfil demográfico y socioeconómico: se encuentran en edad productiva y reproductiva, se identifican como heterosexuales, son solteras y pertenecen a estratos socioeconómicos bajos.

En relación con lo anterior, la resocialización es un proceso en el que a una persona se le enseñan nuevas normas, valores y prácticas que fomentan su transición de un rol social a otro, lo cual puede implicar formas de cambio menores y mayores y puede ser voluntaria o involuntaria, que va de la mano del acompañamiento continuo de las instituciones. Este proceso es definitivo en la condición de pospenado, pues es de donde el ciudadano que era PPL toma elementos para incluirse de forma activa en la sociedad, siendo los procesos de formación en centros penitenciarios los espacios propicios donde se deben dar estas herramientas.

Ahora bien, frente a los modelos pedagógicos en centros penitenciarios, Del Pozo y Añaños (2012) exponen el término de modelo socioeducativo emergente que ha sido implementado en contextos carcelarios porque su enfoque humanista y crítico de las dimensiones epistemológicas de los modelos pedagógicos racionales, genera la posibilidad de adaptar las estrategias, categorías, estructuras y ambientes a las realidades penitenciarias. Los hallazgos de la investigación señalan que es imprescindible la compañía y continuo asesoramiento en los procesos de reconstrucción multidimensional de las personas privadas de la libertad, específicamente en cuanto a la exclusión y la transición hacia la libertad.

En cuanto a lo anterior, el Modelo Educativo para el Sistema Nacional Penitenciario y Carcelario. Una Opción por una experiencia Educativa desde la Investigación, la Resignificación de la Existencia y la Articulación Escuela - Vida (INPEC, 2019), tiene como principios que a través de su existencia el hombre desarrolla dos tipos de actividades: una objetiva y otra subjetiva.

De acuerdo con este planteamiento, este modelo pedagógico se basa en los principios de que el ser humano es un ser inconcluso, en permanente desarrollo humanístico e histórico; sobre la naturaleza humana se dan las relaciones sociales e históricas y los procesos de simbolización colectivos 
e institucionales que se generan sobre las mismas; por lo tanto, el ser humano es de naturaleza biopsicosocial, histórico y cultural. Desde la conciencia social del ser humano se desarrolla la reconstrucción y resignificación teórica de la praxis individual y colectiva; esta construcción simbólica ocurre por procesos de interacción e interiorización consciente de las actividades de las que es actor colectivo y protagonista individual.

En el mismo escenario, los principios del PEl indican que la cotidianidad se convierte en un campo de saber vivencial a través de la construcción y deconstrucción de los objetos de conocimiento, en un proceso que devela intencionalidades y problematizaciones plasmadas en una mediación pedagógica, para producir saberes elaborados transdisciplinariamente, donde la resignificación se da como actividades de simbolización significativas de las vivencias con diversos niveles de complejidad, en los significados que van desde lo ontológico hasta lo epistemológico.

Es así como esta resignificación se da sobre propiedades, cualidades o aspectos lógicos fundamentales tales como el funcionamiento, la estructura, la esencia, la tendencia, el génesis y el devenir; por ende, la producción de saberes se da en la actividad social educativa, donde juega un papel fundamental el estudiante como actor en la toma de decisiones sobre el rumbo de su vida en el contexto donde está ubicado. Para concluir, el PEI determina que el saber implica un saber pensar, o sea, saber producir saber, realizarlo y utilizarlo para su transformación y resignificación y esto conlleva a un saber ser, es decir, quién soy, de dónde vengo y para dónde voy, como proyecto de vida.

\section{Metodología}

En este proyecto de investigación se realizó una sistematización de experiencia en el campo de la educación penitenciaria para visibilizar y reflexionar sobre los impactos del proyecto educativo del INPEC de un grupo de mujeres pospenadas del Establecimiento Penitenciario de Mediana Seguridad y Carcelario de Neiva- EPMSC. De acuerdo con Jara (2012), la sistematización es un ejercicio de producción de conocimiento crítico desde la práctica, que se ha aplicado en la investigación educativa porque permite la reflexión acerca de un fenómeno determinado, a través del interés por aprender de la experiencia, sensibilidad para dejar hablar la experiencia por sí misma y la habilidad para hacer análisis y síntesis. 
Se propuso realizar cinco pasos que se adaptaron en este proceso de investigación así: partir de la propia práctica; en un segundo paso se inicia propiamente la sistematización, respondiendo a interrogantes que no tienen secuencia, pero que precisan ser respondidas y que se expusieron en el planteamiento del problema en un apartado anterior; ¿para qué queremos sistematizar?, ¿qué experiencia(s) queremos sistematizar?, ¿qué aspectos centrales de esa(s) experiencia(s) nos interesa sistematizar; en un tercer paso se hace la reconstrucción del proceso vivido; en un cuarto paso se da la reflexión de fondo sobre ¿por qué pasó lo que pasó?, es una interpretación crítica del proceso vivido, más allá de lo descriptivo que trata de encontrar la razón de ser de lo que sucedió en el proceso de la experiencia; en un quinto paso se generan algunos puntos de llegada; es el último tiempo de la sistematización de experiencias como propuesta metodológica, donde toda la reflexión debe dar por resultado la formulación de conclusiones, tanto teóricas como prácticas.

Se enfocó el tipo de investigación a través del paradigma sociocrítico que se caracteriza por una continua acción-reflexión-acción, que implica, en palabras de Ramos (2015) "La búsqueda de transformación social que se basa en la participación, intervención y colaboración desde la reflexión personal crítica en la acción" (p. 13).

La muestra se definió a conveniencia del investigador porque en el entorno laboral se tiene acceso; son 7 mujeres pospenadas de entre 21 y 60 años de edad, con hijos menores de edad, que cumplieron condena en el Establecimiento Penitenciario Carcelario de Mediana Seguridad de Neiva- EPCMS, entre 19 y 108 meses por tráfico, fabricación o porte de estupefacientes, extorsión, concierto para delinquir o porte ilegal de armas y municiones, que participaron del proyecto de TEE, asistiendo a los CLEIS que se desarrollan al interior de la institución. Además de 4 monitoras que son PPL que prestan el servicio de enseñanza por el perfil que tienen luego de un estudio que las clasifica como aptas para el cargo de ofrecer conocimientos como docentes, de entre 21 y 35 años, sindicadas por delitos como concierto para delinquir agravado y tráfico, porte o fabricación de estupefacientes, homicidio o concierto para delinquir y trafico porte y enriquecimiento ilícito.

Se incluyó en la muestra también a los directivos del Establecimiento Penitenciario y Carcelario de Mediana Seguridad de Neiva - EPCMS, 
Capitán, director del EPMSC de Neiva; actualmente lleva 18 años de servicio en la institución, es profesional en administrador de empresas; Teniente, coordinador del área de tratamiento y desarrollo penitenciario; actualmente lleva 24 años de servicio; en el área de tratamiento como coordinador, 11; es profesional en ingeniera industrial y psicología comunitaria; coordinadora del área de educación, Ileva 16 años trabajando con el INPEC, profesional en licenciatura en educación para la democracia, especialista en resolución de conflictos.

Las técnicas e instrumentos de recolección de información que se utilizaron fueron: la entrevista semiestructurada, diarios de campo y la revisión documental. La entrevista se aplicó en dos fases con el propósito de confirmar y afianzar los resultados observados para propiciar un acercamiento con esta investigación. La información se recolectó mediante la grabación de videos, fichas de observación, elaboración de protocolos de entrevistas y grabaciones de voz para cada una.

\section{Resultados}

En este apartado se desarrolló el proceso de análisis e interpretación de la información obtenida mediante la aplicación de técnicas e instrumentos para la recolección de la información; entrevista semiestructurada, la observación no participante y el análisis documental, adecuados para esta investigación. En un sentido más amplio, se pretende mostrar los hallazgos de esta investigación para la categoría procesos pedagógicos que buscó indagar sobre las intencionalidades y propósitos del Proyecto Educativo Institucional del Instituto Nacional Penitenciario. Los aprendizajes generados aportan un impacto significativo de los CLEIS en las PPL, cuando en voz propia expresan que han encontrado ambientes de aceptación, donde han adquirido conocimientos básicos para la vida, pero también han desarrollado habilidades como escribir y leer; otras PPL han logrado cursar el ciclo de educación formal y estos son logros que transforman vidas.

Si contemplamos y reflexionamos acerca del sentido de esta investigación que es revisar los contextos educativos que se han abierto en las instituciones penitenciarias, uno se da cuenta de que los lineamientos del Ministerio Educación Nacional y las teorías múltiples sobre pedagogía, ambientes de aprendizaje y didácticas no se están cumpliendo. No se 
está generando un espacio propicio para que las PPL puedan diferenciar el cumplimiento de la pena en las áreas designadas de reclusión con respecto a los espacios de aprendizaje de los CLEIS y eso, digamos, que no se puede esperar de estos cursos para crear un cambio significativo emocional, físico, cognitivo o mental en los PPL para que cuando cumplan la condena puedan renovar su proyecto de vida.

De ahí que entender la educación en centros penitenciarios más como un espacio donde la PPL pueda cambiar el estrés de la condena por tiempo de capacitación buscando cambiar sus horizontes, se relaciona con el concepto de educación. López (2007) dice que es algo complejo y limitado, donde se entiende esta noción como una modelación de la persona a partir de una cultura y de unas estructuras sociales que propician alcanzar las habilidades que posee cada individuo, reafirmando el poder trasformador de la educación.

De otro lado, para la categoría procesos administrativos se identificó que este aspecto está condicionado por tantos aspectos diversos, como juzgar desde la acción y no desde la intención a aquellos funcionarios que han manifestado ser partidarios de mejorar la calidad de los PPL, pero que en la realidad se ven conmocionados por otro tipo de situaciones prioritarias que requieren soluciones inmediatas que les permitan preservar la vida de los PPL.

Acorde con lo expuesto, el trabajo de los administradores de prisiones es asegurar que los prisioneros sean alimentados, vestidos y protegidos. Los estándares y normas internacionales exigen la satisfacción de las necesidades básicas de prisioneros; no sugieren que los presos deberían ser mejor tratados que la población no reclusa, no delimitan actividades que disminuyan las reincidencias en la pos-pena, no describen que los PPL deben recibir oportunidades para redescubrirse y cambiar sus caminos. Según la experiencia generada en esta investigación, los administradores de centros carcelarios interactúan con mandos administrativos, pero también con la comunidad para explicar que los PPL merecen un trato justo, humano y que los tratos degradantes o la estigmatización no forma parte del castigo por el delito cometido.

Finalmente, los resultados de la categoría proyección social permitieron reflexionar sobre la realidad de una persona que comete un delito en 
Colombia, quien al recobrar su libertad se ve abocada ante una sociedad sin oportunidades, puesto que las personas tienden a señalar y juzgar; ello genera la creación de un estilo de vida con formas de comunicación impregnadas de rasgos de inseguridad, timidez, estigmatización. Al estar recluído en un instituto penitenciario, la pérdida de noción e interacción con el mundo real puede cambiar las habilidades emocionales, cognitivas, de labor y las sociales.

Por ende, es fundamental que en los espacios donde se cumplen las condenas por delitos cometidos, los PPL accedan a espacios que reencaucen las perspectivas de vida que los condujeron a esta situación; es necesario generar programas que las ayuden a encontrar formas de vivir en sociedad siendo productivas para su familia y para la comunidad. Los importantes beneficios personales de la educación en la prisión incluyen mayores ingresos personales, menor desempleo, mayor participación política y comunitaria del pospenado, además de representar mejoras en el bienestar de las personas que forman su núcleo familiar.

Probablemente no sea sorprendente que los trabajos para pospenados sean difíciles de conseguir, pero para las mujeres pospenadas, las barreras al empleo a menudo pueden parecer insuperables. La pobreza, la falta de apoyo para el cuidado de los niños, la poca cualificación para el trabajo, la falta de vivienda y el abuso doméstico pueden dificultar que las mujeres obtengan el apoyo necesario para acceder al trabajo que ayude a mejorar la calidad de vida de sus familias.

\section{Conclusiones}

En cuanto al objetivo, se dio la tarea de sistematizar una experiencia en el campo de la educación penitenciaria para visibilizar y reflexionar sobre los impactos del proyecto educativo del INPEC en la cualificación de un grupo de mujeres pospenadas. Como docente, como funcionario del INPEC y como mujer se enfrentan procesos reflexivos que conjugan las limitaciones de los espacios carcelarios, con las características individuales de cada mujer PPL, sumado a las condiciones que la enorme brecha social que se ha tejido en Colombia a lo largo de las décadas; no obstante, es posible manifestar que se logró este objetivo de investigación. 
Es un proceso que permitió percibir a las PPL mujeres a través de las particularidades de cada uno de sus casos, que tiene características, causas y efectos diferentes, y ver una realidad social consolidada donde la mujer colombiana sigue sufriendo la inequidad de género que se construyó ancestralmente en la sociedad. La mujer por su condición de género en Colombia es vulnerable, se le sigue estigmatizando por su feminidad, sufre abuso, no recibe las mismas oportunidades que el hombre y tiene que crecer bajo direccionamientos patriarcales que limitan el desarrollo de su potencial como ser humano.

De ahí que sobre el objetivo de indagar sobre las intencionalidades y propósitos del Proyecto Educativo Institucional del Instituto Nacional Penitenciario en una práctica concebida para la población de internos del Establecimiento Penitenciario de Mediana Seguridad y Carcelario de Neiva- EPMSC, se logró al reconocer particularidades de las clases en los CLEIS que sugieren una seria diferenciación entre el servicio educativo que se les da a los hombre y el que se les da a las mujeres: no se le brindan las mismas condiciones a los hombres y a las mujeres para que se eduquen.

Dicho lo anterior, aunque el PEI no está dirigido por tanto a la afectación de los internos únicamente, sino a movilizar todo el aparato institucional para desarrollar nuevas formas de comprender el modo como socialmente se constituyen sujetos, se debe percibir el contexto educativo penitenciario con rasgos que difieren de los entornos educativos formales. En consecuencia, con respecto a otro de los objetivos de investigación al querer reconocer los impactos del proyecto de rehabilitación social para la población de internas del Establecimiento Penitenciario de Mediana Seguridad y Carcelario de Neiva- EPMSC en un grupo de mujeres pospenadas, se decidió observar a las estudiantes para identificar si el espacio era generador de nuevas perspectivas o, por el contrario, remarcaba la ya tediosa situación de privación de la libertad y en expresiones de cada entrevistado independiente de la posición que ocupó en el proceso; siendo estudiante, guiando como profesor o apoyando como dirigente, recalcaron que los programas educativos sí sirven, son un espacio que libera en la reclusión y que bien conducido cambia la perspectiva de vida de la persona. En relación con lo anterior, la resocialización es un proceso en el que a una persona se le enseñan nuevas normas, valores y prácticas que fomentan su transición de un rol social a otro y esto puede implicar formas de cambio. 
Con respecto al último objetivo de investigación, es posible decir que la sistematización de experiencias en el campo de la educación penitenciaria permite visibilizar el impacto del proyecto educativo del INPEC en la cualificación y resignificación de las potencialidades de un grupo de mujeres pospenadas, siendo un método que permite desde el sentir, el ver, el oler la generación de reflexiones de vida que potencian la habilidad del investigador al reconocer que en cada momento se puede aprender, se puede analizar, se experimenta pero sobre todo se construye conocimiento, que puede o no impactar los contextos en los que está inmersa esta búsqueda de espacios investigativos. Y, definitivamente, para los entornos educativos aporta desde las formas de pensamiento complejo métodos para responder a las necesidades especificadas de cada grupo académico.

\section{Referencias Bibliográficas}

Del Pozo, J., Añaños, F. (2012). La Educación Social Penitenciaria: ¿De dónde venimos y hacia dónde vamos?. Recuperado de: https://pdfs.semanticscholar. org/82db/89b0dd289b489b8813a0574b3100805db706.pdf.

Español, S.W.F., Moreno, F.B. (2014). La educación en cárceles, una experiencia desde un establecimiento de reclusión. Hojas y Hablas, 11, 7-19.

Instituto Nacional Penitenciario -INPEC. (2019). Informe y boletines estadísticos. Recuperado de: https://www.inpec.gov.co/estadisticas/-/document_library/ TWBuJQCWH6KV/view/49294.

Instituto Nacional Penitenciario -INPEC. (2019). Modelo educativo para el sistema penitenciario y carcelario colombiano. Bogotá: INPEC.

Jara, O. (2012). Sistematización de experiencias, investigación y evaluación: aproximaciones desde tres ángulos. Revista Internacional sobre Investigación en Educación Global y para el Desarrollo, 1, 56- 70.

Jara, O. (2019). La sistematización de experiencias: nuevas rutas para el quehacer académico en las universidades. Medellín; Divegráficas.

López, G. I. (2007). Co- construyendo un nuevo paradigma que haga emerger la vida: educación existencial, autobiografía y método. Revista Diálogos. Educación y formación de personas adultas, 1(2), 23-34.

Ramos, C. (2015). Los paradigmas de la investigación científica. Av. psicol, 23(1), 9-17.

Rosas, C, E. (2017). Análisis del modelo educativo implementado por el INPEC en la reclusión de mujeres de Bogotá, (Tesis de especialización). Bogotá; Universidad Militar Nueva Granada. 
Sánchez, A., Rodríguez, L., Fondevila, G., Morad, J. (2018). Mujeres y prisión en Colombia, desafios para la política criminal desde un enfoque de género. Bogotá; Fundación Cultural Javeriana de Artes Gráficas - Javegraf.

Schneider, G., (2018). De la educación en cárceles de Argentina y España. Entre el enfoque de derechos y el tratamiento penal. Revista de Estudios Marítimos y Sociales, 1, 10-23. 